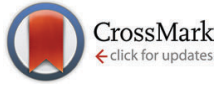

Cite this: Phys. Chem. Chem. Phys., 2014, 16, 21953

Received 29th July 2014, Accepted 1st September 2014

DOI: $10.1039 / \mathrm{c} 4 \mathrm{cp} 03369 \mathrm{~h}$

www.rsc.org/pccp

\section{Surface core-level binding energy shifts for $\mathrm{MgO}(100) \dagger$}

\author{
Connie J. Nelin, ${ }^{a}$ Felix Uhl, ${ }^{b}$ Volker Staemmler, ${ }^{b}$ Paul S. Bagus, ${ }^{* c}$ Yuichi Fujimori, ${ }^{d}$ \\ Martin Sterrer, ${ }^{d}$ Helmut Kuhlenbeck ${ }^{d}$ and Hans-Joachim Freund ${ }^{d}$
}

Theoretical and experimental results for the surface core-level binding energy, $\mathrm{BE}$, shifts, SCLS, for $\mathrm{MgO}(100)$ are presented and the anomalous $\mathrm{O}(1 \mathrm{~s})$ SCLS is interpreted in terms of the surface electronic structure. While the $M g(2 p)$ surface $B E$ shifts to a higher value than bulk by $\approx 1 \mathrm{eV}$ as expected from the different surface and bulk Madelung potentials, the $O(1 \mathrm{~s}) \mathrm{SCLS}$ is almost 0 rather than $\approx-1 \mathrm{eV}$, expected from the Madelung potentials. The distortion of the surface atoms from the spherical symmetry of the bulk $\mathrm{Mg}$ and $\mathrm{O}$ atoms is examined by a novel theoretical procedure. The anomalous O SCLS is shown to arise from the increase of the effective size of surface $O$ anions.

There have been extensive studies of the surface core-level shifts, SCLS's, for the binding energies, BE's, of metal surfaces and the shifts have been interpreted in terms of differences of the electronic structure and environment of surface and bulk atoms. An early review of SCLS for metal surfaces was made by Egelhoff $^{1}$ and the SCLS was also discussed by Bagus et al. ${ }^{2}$ There have been a large number of interpretations of the SCLS at metal surfaces proposed ranging from the Born-Haber cycle analysis $^{3}$ to a bond-order-length-strength analysis. ${ }^{4}$ However, the SCLS of ionic crystals have not been studied previously. In the present work, we show that the SCLS for oxides may be a means of providing new and novel insights into the electronic structure of these systems.

We have examined an ideal ionic insulator, $\mathrm{MgO},{ }^{2,5}$ in order to identify the essential features responsible for the origin of the SCLS of ionic compounds. These features show that the SCLS for oxides can provide important insight into the properties of oxides. If we consider the bulk and surface atoms to be ideal ions, then the SCLS is given by the difference of the Madelung

\footnotetext{
${ }^{a}$ Consultant, Austin, TX 78730, USA

${ }^{b}$ Theoretische Chemie, Ruhr-Universität Bochum, 44780 Bochum, Germany

${ }^{c}$ Department of Chemistry, University of North Texas, Denton, TX 76203-5017, USA.E-mail: bagus@unt.edu

${ }^{d}$ Department of Chemical Physics, Fritz-Haber-Institut der Max-Planck-Gesellschaft, Faradayweg 4-6, 14195 Berlin, Germany

$\dagger$ Electronic supplementary information (ESI) available: Details of calculations and experimental procedures. See DOI: 10.1039/c4cp03369h
}

potentials of these atoms. For this electrostatic potential effect, the sign and magnitude of the SCLS for the (100) face of an octahedral oxide follow from the fact a bulk ion has 6 nearest counter ion neighbours while a surface ion has only 5 such neighbours. Thus, the magnitude of the surface Madelung potential will be smaller than the magnitude of the bulk Madelung potential. Since the Madelung potential lowers cation and raises anion core-level BE's, ${ }^{6}$ the BE's of a surface cation will be larger than those of a bulk cation, SCLS $>0$, while the BE's of a surface anion will be lower than those of a bulk anion, SCLS $<0$. A direct calculation of the Ewald sums gives an Mg SCLS of $+0.9 \mathrm{eV}$ and an O SCLS of $-0.9 \mathrm{eV}$. Our theory and our experiments do not show the symmetry predicted from these electrostatic considerations. While we find the expected $\mathrm{Mg}$ SCLS of $\approx+1 \mathrm{eV}$, the O SCLS is $\approx 0$ rather than $\approx-1 \mathrm{eV}$. We show that the anomalous O SCLS arises from changes of the charge density, $\rho$, of surface $\mathrm{O}$ anions. In particular, we are able to characterize these changes in terms of changes in the size and distortion from the spherical symmetry of the bulk $\mathrm{O}$ anions. This analysis provides important clarification of commonly held views of the spill out of surface anion charge. We provide strong evidence that it is necessary to take account of a pull back of the anion charge below the surface, which may have important implications for the catalytic activity of oxide surfaces and thin films.

In order to establish that our theoretical results correctly described the bulk and surface electronic structure, we used a large number of materials models and computational methods to determine the $\mathrm{MgO}(100)$ SCLS. We used several embedded clusters and several slab models to describe the bulk and the (100) surface of MgO. The BE's for these materials models were determined with both Hartree-Fock, HF, Self-Consistent Field methods ${ }^{7}$ and with correlated methods capable of determining absolute BE's, with high accuracy. ${ }^{8}$ We also used a number of different basis sets to describe the orbitals used in the cluster wavefunctions, WF's, including large basis sets able to give HF limit results. With all reasonably flexible basis sets, we found similar results for the $\mathrm{Mg}$ and O SCLS with all models; in all 
cases, an anomalously small O(1s) SCLS was found. We present results for a representative case with large clusters and large basis sets, as described below and in the ESI. $\dagger$ Our conclusions from the theory would not change if other large computational models were considered. We have used HF wavefunctions since they allow, through the use of Koopmans theorem, KT, to rigorously separate initial and final state effects. The separation of initial and final state effects is less rigorous with other methods. $^{2}$ The separation is important since the initial state contributions reflect the difference of the surface and bulk atom environments and charge distributions, while the final state contributions are consequences of the different response, or core-hole screening, of surface and bulk atoms. ${ }^{2}$ We restrict ourselves to describing the $\mathrm{HF}$ results for two pairs of embedded clusters. For each pair, one cluster describes the BE's of a bulk atom and the other, the BE's of a surface atom; one of the pairs is used to determine the Mg SCLS and the other to determine the O SCLS. The pair of clusters for the O SCLS are $\mathrm{OMg}_{6} \mathrm{O}_{18} \mathrm{Mg}_{38}$ for the bulk, and $\mathrm{OMg}_{5} \mathrm{O}_{13} \mathrm{Mg}_{25}$ for the surface $\mathrm{BE}$ 's, where the indices in the cluster nomenclature indicate the numbers of atoms in each shell around the central $\mathrm{O}$ atom. The pair of clusters for the $\mathrm{Mg}$ SCLS are $\mathrm{MgO}_{6} \mathrm{Mg}_{18}$ and $\mathrm{MgO}_{5} \mathrm{Mg}_{13}$ for the bulk and surface BE's, respectively. The clusters are embedded in Evjen charges $^{9}$ to represent the Madelung potential. The logic of the choice of these clusters is that the atoms terminating the cluster are all compact $\mathrm{Mg}$ cations rather than polarizable $\mathrm{O}$ anions, important because the terminating $\mathrm{Mg}$ cations provide a compressional effect on the interior $\mathrm{O}$ anions. ${ }^{10}$ HF WF's were calculated for the ground states and for $\mathrm{O}$ and $\mathrm{Mg}$ core-holes on the central atom in the cluster. Details of the clusters, the basis sets, and the calculation of the WF's are given in the ESI. $\dagger$ For $\mathrm{O}$, only $\mathrm{O}(1 \mathrm{~s})$ core-holes are considered since this is the only true O core-level. For $\mathrm{Mg}$, we discuss only the SCLS for the $\mathrm{Mg}(2 \mathrm{p})$ core-holes since this is the level for which X-ray photoelectron spectroscopy, XPS, measurements were made. However, very similar SCLS's were obtained for the other $\mathrm{Mg}$ core-levels. Initially, we consider an ideal unreconstructed $\mathrm{MgO}(100)$ surface since the essential physics of the SCLS is already present for an ideal surface. We then consider models for the corrugation of the surface and examine how the corrugation contributes to the SCLS; while this contribution is small, it is not entirely negligible.

As we noted, the different bulk and surface Madelung potentials lead to SCLS's of $\pm 0.9 \mathrm{eV}$. This is purely an initial state effect since orbital relaxation in the presence of core-holes is not taken into account and, further, it assumes an ideal ionic crystal. For the embedded cluster models, we have determined both initial state, KT, and $\triangle$ SCF BE's. ${ }^{2}$ The $\triangle$ SCF BE's are obtained from the difference of variational total energies optimized separately for the initial and final states and include final state relaxation. ${ }^{2}$ The $\mathrm{Mg}(2 \mathrm{p})$ and $\mathrm{O}(1 \mathrm{~s})$ SCLS obtained using the KT and $\triangle$ SCF BE's are given in Table 1 . The KT and $\triangle$ SCF SCLS differ by only $\approx 0.1 \mathrm{eV}$ even though the total relaxation energy, ${ }^{2} E_{\mathrm{R}}$, is $\approx 20 \mathrm{eV}$ for $\mathrm{O}(1 \mathrm{~s})$ and $\approx 5 \mathrm{eV}$ for $\mathrm{Mg}(2 \mathrm{p})$, indicating that initial state effects dominate to determine the SCLS. The differential $E_{\mathrm{R}}$ between surface and bulk is $+0.13 \mathrm{eV}$ for $\mathrm{Mg}(2 \mathrm{p})$ indicating a larger $E_{\mathrm{R}}$ for the bulk $\mathrm{Mg}$ atom, hardly surprising since the bulk atom has
Table 1 KT and $\triangle$ SCF SCLS, in eV, for Mg(2p) and O(1s) XPS

\begin{tabular}{lll}
\hline & $M g(2 \mathrm{p})$ & $\mathrm{O}(1 \mathrm{~s})$ \\
\hline $\mathrm{KT}$ & +0.81 & +0.19 \\
$\Delta \mathrm{SCF}$ & +0.94 & +0.04 \\
\hline
\end{tabular}

more $\mathrm{O}$ anion neighbours than a surface atom and, hence, greater relaxation energy. The differential $E_{\mathrm{R}}$ is $-0.15 \mathrm{eV}$ for $\mathrm{O}(1 \mathrm{~s})$ ionization indicating a larger relaxation for the surface $\mathrm{O}$ anion, again hardly surprising since the surface $\mathrm{O}$ has more freedom to distort than the constrained bulk atom. ${ }^{10}$ The critical point is that the SCLS is close to the expected electrostatic value for the $\mathrm{Mg} \mathrm{BE}$ but almost 0 for the $\mathrm{O} \mathrm{BE}$.

The bulk and surface BE's, measured with un-monochromated $\mathrm{Al} \mathrm{K} \alpha \mathrm{X}$-Rays for thick, 30 monolayer, MgO films grown on $\mathrm{Mo}(100)$, are shown in Fig. 1. The Mo substrate allowed us to anneal the $\mathrm{MgO}$ film to remove defects and adsorbates. The measurements were made for electron exit angles of $0^{\circ}$, or normal exit, which is most bulk sensitive, and $80^{\circ}$, or grazing exit, which is most surface sensitive. The experimental data has been fit with Voigt-type functions adjusted to give the best fit to the XPS data. Details of the XPS measurements are given in the ESI. $\dagger$ Our analysis of the XPS data, described in the ESI, $\dagger$ should give the BE's presented in Fig. 1 reliable to the number of figures shown. For the $\mathrm{Mg}(2 \mathrm{p})$ XPS two peaks, corresponding to surface and bulk ionization, were needed while for the $\mathrm{O}(1 \mathrm{~s}) \mathrm{XPS}$, the data could be fit with only a single peak since the surface and bulk peaks could not be resolved. The conclusion from the XPS data is that the Mg SCLS is large, $+0.65 \mathrm{eV}$, and the $\mathrm{O}(1 \mathrm{~s})$ SCLS is too small to be resolved. This is consistent with the theoretical predictions although the measured $\mathrm{Mg}(2 \mathrm{p})$ SCLS is somewhat smaller than the predicted value from our $\mathrm{MgO}$ cluster models. Later, we present evidence that surface corrugation may contribute $\approx 0.1 \mathrm{eV}$ to reducing the $\mathrm{Mg}(2 \mathrm{p})$ SCLS of the perfect $\mathrm{MgO}(100)$ surface, thus, reducing the difference between the theoretical and experimental $\mathrm{Mg}(2 \mathrm{p})$ SCLS. The critical fact is that theory and experiment agree fully
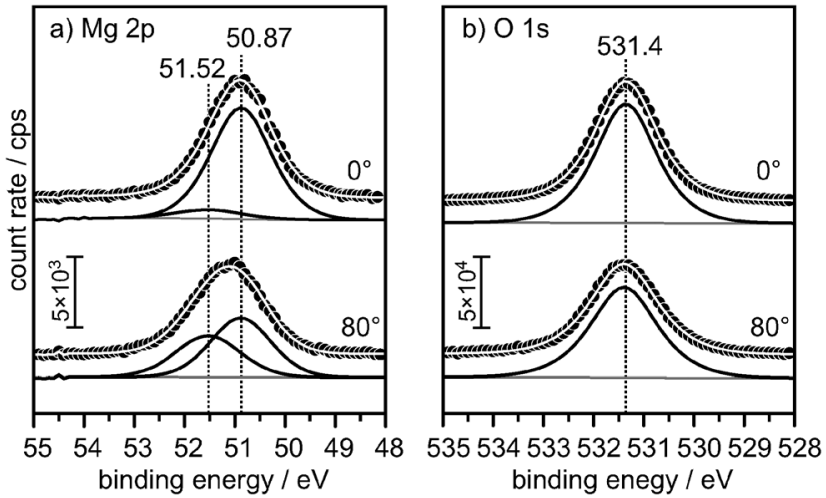

Fig. 1 XPS data for a 30 monolayer $\mathrm{MgO}$ film on $\mathrm{Mo}(100)$ for (a) $\mathrm{Mg}(2 \mathrm{p})$ and (b) $\mathrm{O}(1 \mathrm{~s})$; results are for $0^{\circ}$, bulk sensitive, and $80^{\circ}$, surface sensitive, electron takeoff angles with respect to the MgO surface normal. Circles: raw XPS data; curves: results of the corresponding fits (black: individual fitting components; grey: background; white: fit sum; the black and grey curves are vertically offset for clarity). 
that the $\operatorname{Mg}(2 \mathrm{p})$ SCLS has a magnitude consistent with the different surface and bulk Madelung potentials while the $\mathrm{O}(1 \mathrm{~s})$ SCLS is small, $\approx 0$.

We consider now, in terms of the $\mathrm{MgO}$ electronic structure, the origin of the small O(1s) SCLS. We have conclusively demonstrated that the SCLS's are dominated by initial state effects. Thus, the anomalous SCLS for the $\mathrm{O}(1 \mathrm{~s}) \mathrm{BE}$ must arise from the change in the charge distribution around the surface $\mathrm{O}$ atom. However, it remains to establish the nature of these charge rearrangements. This is done by using the corresponding orbital formalism ${ }^{11,12}$ which allows us to identify the cluster orbital that is most like the orbital of a suitable isolated atomic ion and to quantify the extent to which the atomic orbital is contained, or occupied, in the cluster wavefunction. ${ }^{13} \mathrm{We}$ analyse how this corresponding orbital is different for a bulk atom and a surface atom. In particular, we examine the centre of charge of the surface orbital along the surface normal, $\langle z\rangle$, and the size, or spatial extent, of the surface and bulk orbitals as given by $\left\langle r^{2}\right\rangle$ and its components, $\left\langle x^{2}\right\rangle,\left\langle y^{2}\right\rangle$, and $\left\langle z^{2}\right\rangle$; the origin for all expectation values is the $\mathrm{Mg}$ or $\mathrm{O}$ nucleus. Our concern is for the frontier $\mathrm{O}^{2-}(2 \mathrm{p})$ and $\mathrm{Mg}^{2+}(2 \mathrm{p})$ orbitals since the s orbitals of the $\mathrm{Mg}$ and $\mathrm{O}$ ions are largely chemically inert. This conclusion is supported from examination of the $\mathrm{s}$ corresponding orbitals. Properties of the bulk and surface $\mathrm{Mg}(2 \mathrm{p})$ corresponding orbitals are given in Table 2 where values are given for $2 \mathrm{p}_{x} \equiv 2 \mathrm{p}_{y}$ and $2 \mathrm{p}_{z}$. The full occupation of the $\mathrm{Mg}(2 \mathrm{p})$ orbitals in the bulk show that $\mathrm{Mg}$ in $\mathrm{MgO}$ is very close to an ideal +2 cation. As required by symmetry, the $\left\langle r^{2}\right\rangle$ are identical for $\mathrm{p}_{x}, \mathrm{p}_{y}$, and $\mathrm{p}_{z}$ and the charge of the filled $\mathrm{p}$ shell is spherically symmetric. As expected for the compact $\mathrm{Mg}^{2+}$, the surface $\mathrm{Mg}$ cation has only very small departures from being spherical. This is seen from the small departures of $\langle z\rangle$ from zero and from the essentially equal values of $\left\langle r^{2}\right\rangle$ for all three components of the surface $\mathrm{Mg} 2 \mathrm{p}$ orbital.

The situation is quite different for the $\mathrm{O}(2 \mathrm{p})$ corresponding orbitals whose properties are given in Table 3 . Here we take the $2 \mathrm{p}$ orbital for isolated $\mathrm{O}^{-}$since this is closer to the size of $\mathrm{O}$ in $\mathrm{MgO}$ as shown by the occupations of these orbitals for the bulk and surface $\mathrm{O}$ anions with values of almost 2 electrons for each orbital, Table 3 . The corresponding $2 \mathrm{p}$ orbital for the bulk $\mathrm{O}$ anion is, by symmetry, spherically symmetric. However, the surface $O$ anion has significant departures from spherical symmetry as well as from the size of the bulk $\mathrm{O}$ anion. There is a net motion of the $\mathrm{O}$ charge downward below the surface, shown by the $\langle z\rangle$. This motion is largest for the $\mathrm{O} \mathrm{p}_{z}$ orbital

Table 2 Comparison of $\mathrm{Mg}(2 \mathrm{p})$ corresponding orbitals for a bulk and a surface Mg cation. Properties are $\langle z\rangle$, in $\AA$, and $\left\langle r^{2}\right\rangle,\left\langle x^{2}\right\rangle,\left\langle y^{2}\right\rangle$, and $\left\langle z^{2}\right\rangle$ in $\AA^{2}$, measuring the distortion of the orbital from spherical. The occupation numbers of the $2 p$ orbitals are labeled Occ. For $2 p_{y}$, the values of $\left\langle x^{2}\right\rangle$ and $\left\langle y^{2}\right\rangle$ must be interchanged from those given for $2 p_{x}$

\begin{tabular}{llllllll}
\hline & & Occ. & $\langle z\rangle$ & $\left\langle r^{2}\right\rangle$ & $\left\langle x^{2}\right\rangle$ & $\left\langle y^{2}\right\rangle$ & $\left\langle z^{2}\right\rangle$ \\
\hline Bulk & $2 \mathrm{p}_{x} / 2 \mathrm{p}_{y}$ & 2.000 & 0 & 0.168 & 0.101 & 0.034 & 0.034 \\
& $2 \mathrm{p}_{z}$ & 2.000 & 0 & 0.168 & 0.034 & 0.034 & 0.101 \\
& & & & & & & \\
Surface & $2 \mathrm{p}_{x} / 2 \mathrm{p}_{y}$ & 2.000 & +0.001 & 0.167 & 0.100 & 0.034 & 0.034 \\
& $2 \mathrm{p}_{z}$ & 2.000 & +0.002 & 0.168 & 0.034 & 0.034 & 0.101
\end{tabular}

Table 3 Comparison of $\mathrm{O}(2 \mathrm{p})$ corresponding orbitals for a bulk and a surface $\bigcirc$ anion; see caption to Table 2

\begin{tabular}{llllllll}
\hline & Occ. & $\langle z\rangle$ & $\left\langle r^{2}\right\rangle$ & $\left\langle x^{2}\right\rangle$ & $\left\langle y^{2}\right\rangle$ & $\left\langle z^{2}\right\rangle$ \\
\hline Bulk & $2 \mathrm{p}_{x} / 2 \mathrm{p}_{y}$ & 1.985 & 0 & 0.902 & 0.540 & 0.181 & 0.181 \\
& $2 \mathrm{p}_{z}$ & 1.985 & 0 & 0.902 & 0.181 & 0.181 & 0.540 \\
& & & & & & & \\
Surface & $2 \mathrm{p}_{x} / 2 \mathrm{p}_{y}$ & 1.985 & -0.006 & 0.910 & 0.584 & 0.183 & 0.179 \\
& $2 \mathrm{p}_{z}$ & 1.986 & -0.020 & 0.927 & 0.192 & 0.192 & 0.544 \\
& & & & & & &
\end{tabular}

which is oriented toward the $\mathrm{Mg}^{2+}$ cation in the second layer, but there is also a modest $\langle z\rangle<0$ for the $O \mathrm{p}_{x}$ and $\mathrm{p}_{y}$ orbitals. Overall, there is a pullback of charge below the surface due largely to the missing $\mathrm{MgO}$ layer above the surface. Of course, because of the compact environment of the near $\mathrm{Mg}$ cations, the pullback cannot be particularly large. The effect of this pull back on the effective size of the $\mathrm{O} p$ orbitals is seen from the $\left\langle r^{2}\right\rangle$ and $\left\langle x_{\mathrm{i}}^{2}\right\rangle$; see Table 3 . There is a slight departure from spherical symmetry with $\left\langle r^{2}\right\rangle$ being largest for $\mathrm{p}_{z}$ reflecting the greater pull back for $\mathrm{p}_{z}$. However, there is also an increase of the values of $\left\langle r^{2}\right\rangle$ for the surface over the bulk atom, probably arising from the pull back of $O$ charge. While the increase in the size of the surface over the bulk $\mathrm{O}(2 \mathrm{p})$ orbitals is small, it accounts for a shift of the $\mathrm{O}(1 \mathrm{~s})$ core-level to higher $\mathrm{BE}$, which outweighs the contribution of the reduced Madelung potential leading to a zero total O(1s) SCLS.

In order to understand how changes in the size of an outermost orbital can affect core-level BE's, we use a simple model developed to explain BE shifts between oxides and metals. ${ }^{6,14} \mathrm{We}$ consider the contribution of the electrons in an outer shell, $\mathrm{n} \ell$, to a core-level $\mathrm{BE}, \mathrm{BE} \_$contrib(n $\ell$ ), as being the potential at the nucleus of the $N$ electrons in the shell, $N(\mathrm{n} \ell)$,

$$
\text { BE_contrib }(\mathrm{n} \ell)=-e^{2} \times N(\mathrm{n} \ell) \times\langle 1 / r\rangle_{\mathrm{n} \ell},
$$

where $\langle 1 / r\rangle_{\mathrm{n} \ell}$ is a good approximation to the exact Coulomb integral between $\mathrm{n} \ell$ and the core orbital since the inner shells are compact. ${ }^{6,14}$ Further approximations are that: $\langle 1 / r\rangle$ is close to $1 /\langle r\rangle ;\langle r\rangle$ is close to $\left\langle r^{2}\right\rangle^{1 / 2}$; and we can take $\left\langle r^{2}\right\rangle$ for the $\mathrm{O} 2 \mathrm{p}$ of a surface $\mathrm{O}$ as the average of $\left\langle r^{2}\right\rangle$ for $2 \mathrm{p}_{x}, 2 \mathrm{p}_{y}$ and $2 \mathrm{p}_{z}$ of the surface $\mathrm{O} 2 \mathrm{p}$ orbital, Table 3 . These approximations are acceptable because our concern is for a qualitative interpretation of the $\mathrm{O}(1 \mathrm{~s})$ SCLS. Using the values of $\left\langle r^{2}\right\rangle$ for the bulk and surface $\mathrm{O}(2 \mathrm{p})$ orbital, we find that the increase in size of the $\mathrm{O}(2 \mathrm{p})$ orbital at the $\mathrm{MgO}(100)$ surface compared to the bulk would lead to an increase in the surface atom $\mathrm{O}(1 \mathrm{~s}) \mathrm{BE}$ of $+0.7 \mathrm{eV}$. This increase almost exactly cancels the decrease in the $\mathrm{O}(1 \mathrm{~s}) \mathrm{BE}$ due to the different Madelung potentials of surface and bulk $\mathrm{O}$ anions.

A theoretical study of the extended periodic $\mathrm{MgO}(100)$ surface $^{15}$ shows that there is a modest corrugation of the $\mathrm{MgO}(100)$ surface. To account for the effects of surface rumpling on the SCLS, we modified our cluster model of a perfect (100) surface. We have displaced the central surface ion in the surface clusters along $z$, the surface normal, and determined the changes in the SCLS, $\triangle$ SCLS, with respect to the SCLS for $z=0$, the ideal $\mathrm{MgO}(100)$ surface. We find the equilibrium position of the $\mathrm{Mg}$ cation is $z \approx-0.06 \AA$ below the surface layer, while the $\mathrm{O}$ anion is displaced $z \approx+0.05 \AA$. These displacements have the same direction as found in the study of 
Table 4 Changes in the SCLS, $\triangle \mathrm{SCLS}$, from the ideal MgO(100) surface as the $\mathrm{Mg}$ and $\mathrm{O}$ ions are displaced along $\mathrm{z}$, with $z=0$ for ideal $\mathrm{MgO}(100)$

\begin{tabular}{llr}
\hline & $\Delta$ SCLS & \\
\cline { 2 - 3 }$z / \AA$ & $\mathrm{Mg}(2 \mathrm{p})$ & $\mathrm{O}(1 \mathrm{~s})$ \\
\hline+0.080 & - & -0.02 \\
+0.053 & - & -0.01 \\
+0.026 & +0.05 & -0.00 \\
0.000 & 0.00 & 0.00 \\
-0.026 & -0.04 & +0.00 \\
-0.053 & -0.09 & \\
-0.080 & -0.12 &
\end{tabular}

Boese and Sauer, ${ }^{15}$ albeit with somewhat larger magnitudes for the rumpling. Since the potential curve for displacement of the surface ions is very shallow, it is difficult to precisely determine the extent of the corrugation which may be sensitive to small changes in the computational parameters. The $\triangle$ SCLS given in Table 4 are for the $\triangle \mathrm{SCF}$ BE's and include the effects of changes in the final state relaxation; however, we found similar changes in the SCLS with corrugation for the KT BE's. The changes in the $\mathrm{O}(1 \mathrm{~s})$ SCLS are very small for the $\mathrm{O}$ displacement; at the maximum $z=+0.08 \AA$ considered, the SCLS differs by $0.02 \mathrm{eV}$ from the perfect surface. The changes in the $\mathrm{Mg}(2 \mathrm{p})$ SCLS for corrugation of the $\mathrm{Mg}$ cation are also small but significantly larger than those for corrugation of $\mathrm{O}$. For the maximum $\mathrm{Mg}$ displacement considered, $z=-0.08 \AA$, the SCLS is reduced by $0.1 \mathrm{eV}$, bringing the theoretical $\mathrm{Mg}(2 \mathrm{p})$ SCLS into closer agreement with the XPS measurements. Thus, the observed Mg SCLS, which is smaller than the value predicted from our cluster model theory for a perfect $\mathrm{Mg}(100)$ surface, provides additional support that $\mathrm{MgO}(100)$ is corrugated. It is possible that an improved model of the surface corrugation that takes into account the simultaneous corrugation of the $\mathrm{Mg}$ cations and the $\mathrm{O}$ anions would further improve the agreement between the theoretical and experiment for the $\mathrm{Mg}(2 \mathrm{p})$ SCLS.

The theoretical and measured SCLS of the $\mathrm{Mg}(2 \mathrm{p})$ XPS show that the SCLS is close to the $+0.9 \mathrm{eV}$ difference of the bulk and surface Madelung potentials, which is the SCLS that is obtained assuming that $\mathrm{MgO}$ is an ideal ionic oxide. On the other hand, the almost 0 SCLS predicted and observed for the O(1s) XPS shows that there must be an effect in the electronic structure of $\mathrm{MgO}$ that cancels the $-0.9 \mathrm{eV}$ SCLS due to the difference of surface and bulk Madelung potentials at $\mathrm{O}$. Our detailed analysis of the charge distribution, $\rho$, of $\mathrm{Mg}^{2+}$ and $\mathrm{O}^{2-}$ in $\mathrm{MgO}$ shows that the surface $\rho\left(\mathrm{Mg}^{2+}\right)$ remains spherically symmetric and has about the same size, or spatial extent, as the bulk $\rho\left(\mathrm{Mg}^{2+}\right)$. On the other hand, $\rho\left(\mathrm{O}^{2-}\right)$ at the surface is distorted from the spherical bulk $\rho\left(\mathrm{O}^{2-}\right)$. There is a pull back of the $\mathrm{O}$ charge below the surface toward the $\mathrm{Mg}^{2+}$ cation directly below the surface $O$ and there is an increase in the size of the surface $\mathrm{O}(2 \mathrm{p})$ electrons compared to the bulk. While the increase in the size of $\rho$ for the surface $O$ is small, it is sufficiently large to offset the contribution of the Madelung potentials toward a smaller surface $\mathrm{O}(1 \mathrm{~s}) \mathrm{BE}$ and to lead to an $\mathrm{O}(1 \mathrm{~s})$ SCLS that is almost 0 . We have examined the effect of surface corrugation of $\mathrm{MgO}(100)$ on the different SCLS for Mg and O. The key findings are that: (1) the $\approx 0 \mathrm{O}(1 \mathrm{~s})$ SCLS is not changed significantly even for relatively large displacements of the $\mathrm{O}$ anion above the surface. And, (2) although the changes of the $\operatorname{Mg}(2 \mathrm{p})$ SCLS with surface corrugation are somewhat larger than those for the $\mathrm{O}(1 \mathrm{~s})$ corrugation, they are also small compared to the total $\mathrm{Mg}(2 \mathrm{p})$ SCLS.

Our results indicate that the SCLS of oxides and other ionic crystals should be explored further to determine their use in terms of characterizing the electronic and geometric structures of these surfaces.

We acknowledge support by the Geosciences Research Program, Office of Basic Energy Sciences, U.S. DOE and by the DFG through their SFB 546, Transition Metal Oxide Aggregates. We thank Helmholtz-Zentrum Berlin for provision of synchrotron radiation for some of the measurements related to the MgO SCLS. Y.F. acknowledges financial support from DAAD and Co. Ltd Takata.

\section{Notes and references}

1 W. F. Egelhoff, Surf. Sci. Rep., 1987, 6, 253.

2 P. S. Bagus, E. S. Ilton and C. J. Nelin, Surf. Sci. Rep., 2013, 68, 273.

3 B. Johansson and N. Martensson, Phys. Rev. B: Condens. Matter Mater. Phys., 1980, 21, 4427.

4 (a) C. Q. Sun, Y. Nie, J. Pan, X. Zhang, S. Z. Ma, Y. Wang and W. Zheng, RSC Adv., 2012, 2, 2377; (b) C. Q. Sun, Phys. Rev. B: Condens. Matter Mater. Phys., 2004, 69, 045105.

5 G. Pacchioni, C. Sousa, F. Illas, F. Parmigiani and P. S. Bagus, Phys. Rev. B: Condens. Matter Mater. Phys., 1993, 48, 11573.

6 P. S. Bagus, G. Pacchioni, C. Sousa, T. Minerva and F. Parmigiani, Chem. Phys. Lett., 1992, 196, 641.

7 I. Levine, Quantum Chemistry, Prentice-Hall, Upper Saddle River, NJ, 2000.

8 F. Uhl and V. Staemmler, J. Phys.: Condens. Matter, 2012, 24, 305501.

9 H. M. Evjen, Phys. Rev., 1932, 39, 675.

10 J. Q. Broughton and P. S. Bagus, Phys. Rev. B: Condens. Matter Mater. Phys., 1987, 36, 2813.

11 H. Ågren and P. S. Bagus, J. Am. Chem. Soc., 1985, 107, 134. 12 A. T. Amos and G. G. Hall, Proc. R. Soc. London, Ser. A, 1961, 263, 483.

13 C. J. Nelin, P. S. Bagus and M. R. Philpott, J. Chem. Phys., 1987, 87, 2170.

14 C. Sousa, T. Minerva, G. Pacchioni, P. S. Bagus and F. Parmigiani, J. Electron Spectrosc. Relat. Phenom., 1993, 63, 189.

15 A. D. Boese and J. Sauer, Phys. Chem. Chem. Phys., 2013, 15, 16481. 